\title{
Prévention et prise en charge des maladies chroniques: une approche applicable en Suisse
}

\section{Peytremann-Bridevaux ${ }^{a}$, B. Burnand ${ }^{b}$}

a Dr Isabelle PeytremannBridevaux, cheffe de clinique, MPH, DSc, FMH Prévention et Santé Publique et Médecine Interne, Institut universitaire de médecine sociale et préventive (IUMSP), Université de Lausanne et Centre hospitalier vaudois (CHUV)

b Professeur Bernard Burnand, $\mathrm{MPH}, \mathrm{FMH}$ Prévention et Santé Publique et Médecine Interne, Institut universitaire de médecine sociale et préventive (IUMSP), Université de Lausanne et Centre hospitalier vaudois (CHUV)

Aucun des deux auteurs ne déclare de conflits d'intérêts.

Isabelle Peytremann-Bridevaux est au bénéfice d'une bourse PROSPER du Fonds National Suisse de la Rechercher Scientifique (subside 32333B-123817)

Correspondance:

Dr Isabelle PeytremannBridevaux

Institut universitaire de médecine sociale et préventive (IUMSP)

Bugnon 17

CH-1005 Lausanne

Isabelle.Peytremann-Bridevaux @chuv.ch

www.iumsp.ch

\section{Résumé}

Les maladies chroniques représentent un fardeau important pour la société, en termes de morbidité, dépendance, qualité de vie, mortalité et coûts de la santé. La prise en charge des maladies chroniques constitue ainsi une priorité des systèmes de santé. Tandis que des programmes de prévention et prise en charge des maladies chroniques («prevention and chronic disease management») ont été mis sur pieds depuis plus d'une décennie d'abord en Amérique du Nord puis en Europe, leur développement en Suisse est récent. Ces programmes sont explicitement structurés et organisés, centrés sur les patients et comportent constamment un élément d'éducation thérapeutique. De plus, ils impliquent un travail en équipe ainsi que des prises en charge stratifiées en fonction de la sévérité de la maladie et des besoins des patients, et basées sur les preuves de leur efficacité. Il est important que les médecins et autres professionnels de la santé, ainsi que tous les autres acteurs du système sanitaire reconnaissent la nécessité de mettre en place des modalités adéquates et efficaces de prise en charge des patients présentant des maladies chroniques, participent à leur développement et les soutiennent.

\section{Contexte général}

L'un des défis auquel le système de santé doit faire face, en Suisse comme ailleurs, est son adaptation insuffisante à la forte prédominance des maladies chroniques. Le modèle actuel est approprié pour les demandes ponctuelles et les urgences, moins pour la prise en charge au long cours de patients ayant une maladie chronique et des besoins multiples. Actuellement, les prises en charge multidisciplinaires sont le plus souvent proposées chez des patients présentant des situations complexes, comme en (psycho-)gériatrie ou diabétologie par exemple. Cependant, de telles prises en charge coordonnées sont rarement mises en route lorsque la situation est moins avancée et leur impact moindre.

En outre, des changements majeurs, qui vont exercer une pression importante sur la médecine de premier recours[1], sont attendus dans la composition du corps médical, suite au remplacement insuffisant des médecins qui vont quitter leurs fonctions au cours des prochaines années et à la modification des modes de pratique de la profession. Des manques similaires affecteront les autres professionnels de santé, particulièrement le personnel infirmier.
Chez les patients présentant une (des) maladie(s) chronique(s), une meilleure répartition des tâches entre le médecin et les autres professionnels de la santé permettrait au médecin de rester disponible pour les aspects de la prise en charge et les situations qui nécessitent son intervention, tout en maintenant le suivi au long cours et la coordination des soins. Des publications suisses récentes soulignent la nécessité d'adapter les pratiques à la prédominance des maladies chroniques et au vieillissement de la population, et d'adopter une collaboration plus rapprochée entre divers professionnels des soins $[2,3]$.

Des programmes de prévention et prise en charge structurée des maladies chroniques, habituellement dénommés «chronic disease prevention and management programs» en anglais, ont été développés et évalués en Amérique du nord et en Europe. Le but de cet article est de présenter cette approche, d'en examiner la justification et l'efficacité, ainsi que d'analyser comment elle pourrait être adaptée en Suisse.

\section{Le fardeau des maladies chroniques}

L'augmentation de l'espérance de vie et de l'espérance de vie en bonne santé, ainsi que le vieillissement de la population, sont principalement responsables de l'augmentation de la prévalence actuelle et à venir des maladies chroniques. Les maladies chroniques, les plus fréquentes étant l'insuffisance cardiaque, le diabète, la dépression, la broncho-pneumopathie chronique obstructive, l'asthme et l'arthrose, représentent un fardeau pour la société, en termes de morbidité, mortalité précoce, dépendance, qualité de vie et coûts de la santé [4]. En effet, en Europe par exemple, les maladies chroniques sont responsables de $88 \%$ des décès [5] et d'une part importante des coûts de la santé [6].

Les estimations pour le canton de Vaud montrent que le vieillissement de la population provoquera une augmentation de la prévalence de l'insuffisance cardiaque, du diabète, de la dépression, des démences et de la broncho-pneumopathie chronique obstructive, entre autres, d'au moins 50\% d'ici 2030 [7]. La prise en charge des maladies chroniques constitue ainsi une priorité des systèmes de santé.

\section{Qu'est-ce que la prévention et prise en charge des maladies chroniques («chronic disease prevention and management»)?}

En 1997, Ellrodt décrivait les programmes de prévention et prise en charge des maladies chroniques comme correspondant à «une approche multidiscipli- 
naire des soins pour les maladies chroniques qui met l'accent sur la coordination et la globalité des soins, sur l'ensemble du spectre de la maladie et du système de santé» [8]. Les définitions sont toutefois nombreuses $[9,10]$, et évoluent dans le sens de l'intégration et de la description explicite des différentes composantes décrites individuellement [11].

La prévention et prise en charge des maladies chroniques est une approche qui consiste à réorganiser les soins de santé et à structurer les prises en charge des patients souffrant de maladies chroniques. Le but est d'éliminer la fragmentation et la discontinuité des soins, et de limiter l'hospitalisation, pour ainsi améliorer la qualité des soins et du suivi des patients. Cette perspective s'inscrit dans une réflexion générale sur l'organisation des systèmes de soins pour les prises en charge des maladies chroniques, dont le "Chronic Care Model» reste le modèle de référence $[12,13]$.

Les programmes de prévention et de prise en charge des maladies chroniques sont centrés sur les patients et leurs besoins, souvent multiples et complexes. Ils comprennent une dimension d'éducation thérapeutique et d'auto-prise en charge de la maladie, nécessitent l'interdisciplinarité et le travail en équipe, impliquent des prises en charge basées sur les preuves de leur efficacité, ainsi que des mesures et évaluations régulières des processus et résultats couplés à des systèmes de retour d'information aux professionnels de santé. En outre, l'intensité de ces programmes doit être stratifiée en fonction de la sévérité de la maladie et des besoins des patients, qui sont répartis en plusieurs catégories. A chaque palier successif, la prise en charge s'intensifie et se complète. Les patients peuvent passer d'un niveau à un autre en fonction de l'évolution de leur maladie et de leurs besoins. Ainsi, chez la majorité (70-80\%) des patients présentant une maladie chronique les symptômes sont stables et le risque de complications et d'hospitalisation est

Figure 1

Stratification de la prise en charge en fonction de la sévérité de la maladie et des besoins des patients.

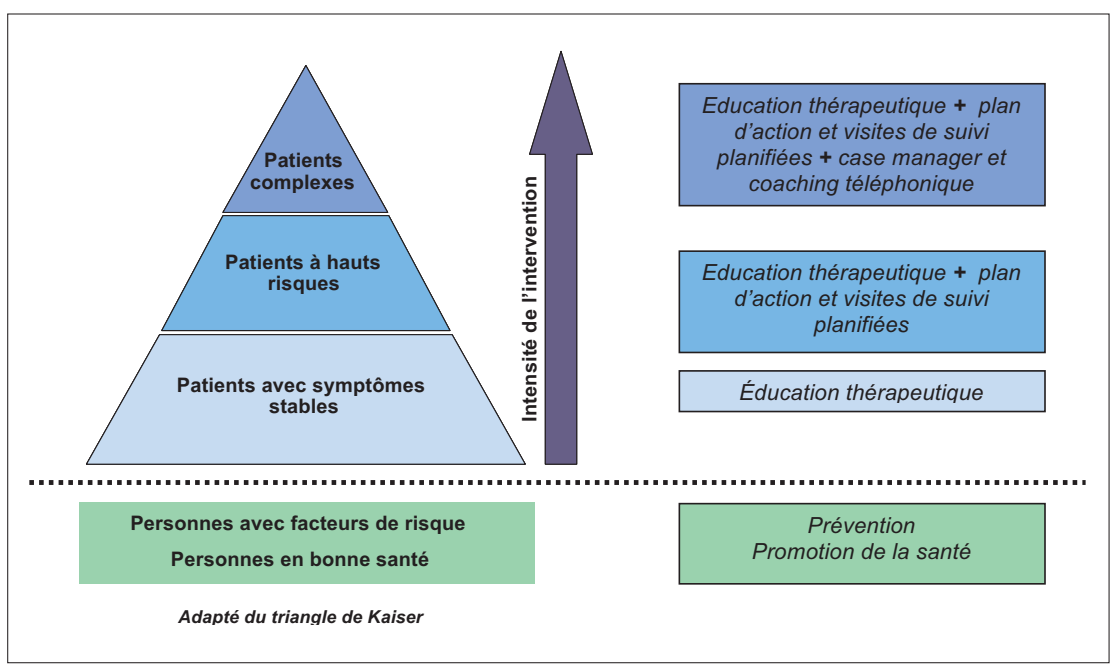

faible. Ils ont surtout besoin d'une aide à l'autoprise en charge; ces patients peuvent être suivis en grande partie par une infirmière spécialisée, dans un objectif d'éducation thérapeutique. Les patients à plus haut risque d'hospitalisation ou de complications nécessitent en plus un suivi plus régulier par le médecin de premier recours, ou occasionnellement le spécialiste. Des plans d'action et des visites de suivi régulières sont proposées. Finalement, les patients «complexes» dont l'état est instable en raison d'une maladie mal contrôlée et/ou de la présence d'autres maladies chroniques requièrent un suivi plus personnalisé, qui implique aussi régulièrement des médecins spécialistes. A ce stade un encadrement téléphonique et un suivi rapproché par un «case manager» peuvent être introduits, par exemple. Le choix des interventions et professionnels décrits à chacun des stades est à adapter au contexte sanitaire local et aux préférences des patients (exemples illustrés dans la figure et les vignettes).

A l'ensemble des patients présentant une (des) maladie(s) chronique(s) s'ajoutent toutes les personnes en bonne santé ou avec facteurs de risque (obésité, tabagisme, hypertension artérielle, sédentarité, ...) pour lesquelles des interventions de santé publique, de prévention et promotion de la santé, mais aussi de prestations individualisées de soins de santé doivent être considérées. Ces interventions ne ciblent pas une maladie particulière, mais l'ensemble des comportements favorables à une bonne santé.

Les maladies chroniques ciblées par les programmes de prévention et prise en charge sont caractérisées par un important fardeau, des bénéfices attendus d'une participation active du patient à la gestion de sa maladie, ainsi qu'une pratique médicale usuelle qui s'écarte souvent, sans justification, des recommandations pour la pratique clinique fondées sur les preuves. Ainsi, le diabète, l'insuffisance cardiaque, les cardiopathies ischémiques, l'asthme, la bronchopneumopathie chronique obstructive (BPCO) et la dépression sont principalement ciblés par ces programmes.

\section{Développement des modèles et programmes de prévention et prise en charge des maladies chroniques}

Initialement développés aux USA par les entreprises pharmaceutiques au début des années 1990 pour augmenter les ventes de médicaments, les programmes de prévention et prise en charge des maladies chroniques («disease management») ont ensuite été utilisés par les entreprises de managed care («managed care organizations»), comme un outil, parmi d'autres tels le passage obligé par le médecin généraliste, l'autorisation préalable pour certaines procédures, les listes restrictives de médicaments, ou divers incitatifs financiers, visant à limiter l'augmentation des coûts de la santé et à améliorer la qualité des soins. Actuellement aux USA, les principaux pourvoyeurs de ce modèle de 
prise en charge des maladies chroniques sont des organismes privés qui vendent leur programmes aux assurances, et des organismes tels Kaiser Permanente ou Group Health Cooperative qui intègrent les fonctions d'assurance et de dispensation des soins, et dont les caractéristiques (participation des médecins aux objectifs de qualité et d'efficience, paiement par capitation, travail en équipe, système d'information performant) permettent une telle approche. Au Canada, depuis le début des années 2000, les gouvernements fédéraux et provinciaux ont établi des politiques et initiatives visant le développement de programmes de prévention et prise en charge des maladies chroniques. Ainsi, principalement sur la base du Chronic Care Model, une variété de stratégies ont été mises sur pied par plusieurs provinces (Colombie Britannique, Ontario, Saskatchewan, Alberta, Québec).

En Europe, des programmes de prise en charge des maladies chroniques se sont développés au cours de la dernière décennie, comme une tentative de réponse aux problèmes médicaux (augmentation du fardeau des maladies chroniques, qualité des soins sub-optimale), organisationnels (fragmentation des soins, va-

\section{Vignettes cliniques}

\section{Patients complexes}

Avec le médecin diabétologue, et les services de soins à domicile, un médecin de premier recours met sur pied un suivi rapproché pour une patiente diabétique de 75 ans, qui présente non seulement des complications rénales et cardiovasculaires de son diabète mais également une hypercholestérolémie et une dépression mineure.

Il est entre autres convenu que l'infirmière spécialisée en diabétologie s'occupe de superviser le monitorage de la glycémie par la patiente et ses ajustements de I'insulinothérapie et de son alimentation, avec notamment un suivi téléphonique $2-3 \times /$ semaine au cours du prochain mois. Le contrôle du diabète reste toutefois difficile. Ainsi, suite à un consensus et selon le désir de la patiente, le médecin diabétologue prendra principalement en charge cette patiente. Le médecin généraliste restera à disposition de celle-ci pour le suivi de ses symptômes dépressifs et autres problèmes de santé potentiels.

Un rendez-vous téléphonique mensuel impliquant le médecin traitant, le diabétologue, l'infirmière spécialisée en diabétologie et l'infirmière responsable du suivi à domicile permettra d'ajuster le traitement et suivi, notamment afin d'éviter une nouvelle hospitalisation.

Patients à hauts risques

Un patient diabétique et tabagique, âgé de 65 ans, est hospitalisé pour un infarctus du myocarde. Au riations régionales des pratiques médicales) et économiques (augmentation des coûts de la santé) $[14,15]$. Les solutions proposées varient en fonction de la volonté politique et institutionnelle de considérer comme prioritaires les maladies chroniques et leur prise en charge, de l'organisation du système de santé et du degré de développement de la médecine de premier recours. Ainsi, les consultations ambulatoires dirigées par des infirmières («nurse-led clinics») se sont considérablement développées en Suède, des casemanagers («community matrons») ont été introduits pour les patients complexes et grands utilisateurs de soins au Royaume-Uni, et divers modèles de prises en charge des maladies chroniques se sont déployés aux Pays-Bas. En Allemagne, une loi sur la gestion de cinq maladies chroniques (diabète, asthme, BPCO, insuffisance cardiaque et cancer du sein), est en vigueur depuis janvier 2003. Son but est non seulement de combler le déficit de qualité des soins des patients souffrant de maladies chroniques, mais aussi d'améliorer la structure de compensation des risques entre assurances. Les assurances sont chargées de créer et mettre en place des programmes de prévention et prise en charge des mala-

décours de cet épisode, son médecin traitant s'assure non seulement que le suivi par l'infirmière spécialisée en diabétologie s'effectue de manière régulière, mais aussi qu'un plan d'action soit discuté et remis au patient. Il s'agit d'améliorer l'autoprise en charge du diabète par le patient, afin de stabiliser la maladie et freiner l'évolution de ses complications.

Ainsi, le patient apprendra à contrôler régulièrement sa glycémie. Sa femme et lui participeront à un cours pratique de cuisine et de diététique. En outre, le patient a arrêté de fumer lors de l'hospitalisation et suite à sa retraite récente, il a décidé d'effectuer une à deux heures de marche quotidienne avec son chien.

\section{Patients avec symptômes stables}

Une femme de 50 ans est traitée par antidiabétiques oraux depuis un an pour un diabète de type II sans complications. Elle ne présente aucune complication et le contrôle de la glycémie a toujours été bon ( $\mathrm{HbA} 1 \mathrm{C}<6,5 \%)$.

Elle a suivi plusieurs séances d'éducation thérapeutique données par une infirmière spécialisée en diabétologie et utilise les brochures d'information qu'elle a reçues.

Des rendez-vous avec son médecin traitant dédiés au suivi du diabète sont organisés régulièrement. Une évaluation de la fonction rénale et un contrôle ophtalmologique ont été effectués, un an après le bilan initial. 
dies chroniques qui doivent remplir un nombre minimum de critères de qualité, dont le contrôle se fait au niveau national. Tandis que les assurances maladie ont intérêt à inclure de nombreux patients puisqu'elles seront financièrement compensées pour le fait d'avoir un patient malade chronique comme client, les médecins et les patients sont incités à participer, par une rémunération spécifique pour les uns, ou une réduction de la participation aux frais médicaux ou de la franchise par exemple pour les autres. En contrepartie, les médecins s'engagent à suivre les recommandations pour la pratique clinique, et les patients à participer aux séances d'éducation thérapeutique, entre autres. La participation du patient est volontaire, mais il est responsable de trouver un médecin acceptant de le suivre (médecin référent) [16,17].

\section{Efficacité et coûts-efficacité des programmes de prévention et prise en charge des maladies chroniques}

L'efficacité des programmes de prévention et prise en charge des maladies chroniques a été évaluée à plusieurs reprises. Les résultats de revues systématiques suggèrent que ces programmes apportent des bénéfices aux patients, en particulier à ceux souffrant d'insuffisance cardiaque ou de diabète. En effet, les risques d'hospitalisation et de mortalité toutes causes sont significativement réduits en cas d'insuffisance cardiaque $[18,19]$, et l'hémoglobine glyquée significativement diminuée $(-0,5 \%)$ chez les patients diabétiques $[20,21]$. Pour les patients avec un diagnostic de $\mathrm{BPCO}$, les études suggèrent une amélioration de la distance de marche et une diminution de l'utilisation des services de santé ainsi qu'un bénéfice en termes de qualité de vie [22]. Les patients dépressifs participant à de tels programmes rapportent moins de symptômes de dépression ainsi qu'une meilleure compliance médicamenteuse [23,24].

Les preuves de l'économicité de ces programmes sont peu nombreuses et ne sont pas consensuelles quant à une réduction de coûts ou à un rapport coûtbénéfice en faveur des programmes évalués. Il ne semble toutefois pas y avoir de preuves d'accroissement des coûts [25-27].

\section{Etat des lieux et perspectives en Suisse}

En Suisse, le développement de programmes de prévention et gestion des maladies chroniques est récent. Cela pourrait s'expliquer par le fait que le système de santé suisse présente des propriétés reconnues comme étant des barrières à ce type de développement, telles que les modalités de financement, par exemple. Néanmoins, quelques initiatives suisses qui comportent un certain nombre de caractéristiques des programmes de prévention et prise en charge des maladies chroniques, ont été récemment identifiées (communication personnelle).

Nous proposons une vision des programmes de prévention et prise en charge des maladies chroniques qui relève d'une approche de santé publique, et tient compte des particularités du système sanitaire, ainsi que des modèles théoriques existants et des expériences réalisées en Suisse et à l'étranger. Ainsi, ces programmes, adaptés au contexte local, s'adresseraient à une communauté, région ou canton, favoriseraient l'accès aux soins de l'ensemble des personnes concernées par les maladies chroniques, et prendraient en compte des approches préventives. Les acteurs comprendraient non seulement les patients et leurs proches, ainsi que les médecins de premier recours et spécialistes, mais aussi les infirmières, pharmaciens, physiothérapeutes, psychologues, diététiciennes, assistants sociaux, etc. provenant tant des structures hospitalières que des soins ambulatoires. L'initiative, la mise en place du cadre de tels programmes et leur suivi reviendraient aux cantons et aux régions, avec l'appui de la confédération. De plus, une participation réelle de tous les acteurs du système de santé, y compris les sociétés cantonales de médecine, les associations professionnelles, les réseaux de soins, les hôpitaux, les services de soins à domicile, les ligues, les associations de patients, entre autres, est nécessaire à la mise en place de tels programmes. Les assureurs doivent être associés à de tels développements, comme partenaires mais non comme promoteurs uniques de ces approches. Des programmes de prévention et gestion des maladies chroniques pourraient se développer dans des régions de taille variable (villes, régions géographiques), mais il faut probablement une taille minimale en terme de nombre de patients porteurs d'un type de maladie chronique pour justifier leur mise en place et assurer leur efficience. Le développement de ces programmes implique non seulement des mesures législatives et contractuelles, mais aussi le soutien à des activités de recherche et de développement, de formation et d'évaluation de programmes ainsi que des actions de sensibilisation et de discussion impliquant la population générale, les patients et les professionnels de santé. Au préalable, il faut que la problématique des maladies chroniques et de leur impact sur la santé de la population et le système de soins de santé, soit reconnue.

\section{Références}

1 Seematter-Bagnoud L, Junod J, Jaccard Ruedin H, Roth M, Foletti C, Santos-Eggimann B. Offre et recours aux soins médicaux ambulatoires en Suisse Projections à l'horizon 2030. Neuchâtel (Suisse); 2008.

2 De GS, Moons P, Callens B, Gut C, Lindpaintner L, Spirig R. Introducing Advanced Practice Nurses / Nurse Practitioners in health care systems: a framework for reflection and analysis. Swiss Med Wkly. 2008; 138:621-8.

3 Giger M, De Geest S. Les soins de premier recours doivent répondre à des exigences rapidement changeantes. De nouveaux modèles de soins et de nouvelles compétences sont nécessaires. Bull Méd Suisses. 2008;89:1839-43. 
4 Mathers CD, Loncar D. Projections of global mortality and burden of disease from 2002 to 2030. PLoS Med. 2006;3:e442.

5 Velasco-Garrido M, Busse R, Hisashige A. Are disease management programmes (DMPs) effective in improving quality of care for people with chronic conditions? Copenhagen; 2003.

6 Hoffman C, Rice D, Sung HY. Persons with chronic conditions. Their prevalence and costs. JAMA. 1996; 276:1473-9.

7 Paccaud F, Peytremann-Bridevaux I, Heiniger M, Seematter-Bagnoud L. Vieillissement: éléments pour une politique de santé publique. Un rapport préparé pour le service de la santé publique du canton de Vaud. Lausanne; 2006.

8 Ellrodt G, Cook DJ, Lee J, Cho M, Hunt D, Weingarten S. Evidence-based disease management. JAMA. 1997; 278:1687-92.

9 DMAA definition of disease management. www dmaa org/dm_definition asp [2007]

10 Ouwens M, Wollersheim H, Hermens R, Hulscher M, Grol R. Integrated care programmes for chronically ill patients: a review of systematic reviews. Int J Qual Health Care. 2005;17:141-6.

11 Schrijvers G. Disease management: a proposal for a new definition. Int J Integr Care. 2009;9:1-2.

12 Epping-Jordan JE, Pruitt SD, Bengoa R, Wagner EH. Improving the quality of health care for chronic conditions. Qual Saf Health Care. 2004;13:299-305.

13 Wagner EH, Austin BT, Davis C, Hindmarsh M, Schaefer J, Bonomi A. Improving chronic illness care: translating evidence into action. Health Aff (Millwood). 2001;20:64-78.

14 Caring for people with chronic conditions. A health system perspective. Maidenhead: Open University Press; 2008.

15 Managing chronic conditions. Experience in eight countries. Copenhagen: European Observatory on Health Systems and Policies \& WHO Regional Office for Europe; 2008.

16 Busse R. Disease management programs in Germany's statutory health insurance system. Health Aff (Millwood). 2004;23:56-67.
17 Greb S, Focke A, Hessel F, Wasem J. Financial incentives for disease management programmes and integrated care in German social health insurance. Health Policy 2006;78:295-305.

18 McAlister FA, Lawson FM, Teo KK, Armstrong PW. A systematic review of randomized trials of disease management programs in heart failure. Am J Med. 2001;110:378-84.

19 Roccaforte R, Demers C, Baldassarre F, Teo KK, Yusuf S. Effectiveness of comprehensive disease management programmes in improving clinical outcomes in heart failure patients. A meta-analysis. Eur J Heart Fail. 2005; 7:1133-44.

20 Knight K, Badamgarav E, Henning JM, Hasselblad V, Gano AD, Jr., Ofman JJ et al. A systematic review of diabetes disease management programs. Am J Manag Care 2005;11:242-50.

21 Norris SL, Nichols PJ, Caspersen CJ, Glasgow RE, Engelgau MM, Jack L, et al. The effectiveness of disease and case management for people with diabetes. A systematic review. Am J Prev Med. 2002;22:15-38.

22 Peytremann-Bridevaux I, Staeger P, Bridevaux PO, Ghali WA, Burnand B. Effectiveness of chronic obstructive pulmonary disease-management programs: systematic review and meta-analysis. Am J Med. 2008; 121:433-43.

23 Badamgarav E, Weingarten SR, Henning JM, Knight K, Hasselblad V, Gano A, Jr., et al. Effectiveness of disease management programs in depression: a systematic review. Am J Psychiatry. 2003;160:2080-90.

24 Neumeyer-Gromen A, Lampert T, Stark K, Kallischnigg $\mathrm{G}$. Disease management programs for depression: a systematic review and meta-analysis of randomized controlled trials. Med Care. 2004; 42:1211-21.

25 Goetzel RZ, Ozminkowski RJ, Villagra VG, Duffy J. Return on investment in disease management: a review. Health Care Financ Rev. 2005;26:1-19.

26 Krause DS. Economic effectiveness of disease management programs: a meta-analysis. Dis Manag. 2005; 8:114-34.

27 Mattke S, Seid M, Ma S. Evidence for the effect of disease management: is $\$ 1$ billion a year a good investment? Am J Manag Care. 2007;13:670-6. 